\title{
Leading-order classical Lagrangians for the nonminimal standard-model extension
}

\author{
J. A. A. S. Reis ${ }^{*}$ and M. Schreck ${ }^{\dagger}$ \\ Departamento de Física, Universidade Federal do Maranhão, 65080-805 São Luís, Maranhão, Brazil
}

(Received 14 December 2017; published 29 March 2018)

\begin{abstract}
In this paper, we derive the general leading-order classical Lagrangian covering all fermion operators of the nonminimal standard-model extension (SME). Such a Lagrangian is considered to be the point-particle analog of the effective field theory description of Lorentz violation that is provided by the SME. At leading order in Lorentz violation, the Lagrangian obtained satisfies the set of five nonlinear equations that govern the map from the field theory to the classical description. This result can be of use for phenomenological studies of classical bodies in gravitational fields.
\end{abstract}

DOI: 10.1103/PhysRevD.97.065019

\section{INTRODUCTION}

Effects from gravitational physics and quantum physics are expected to be on an equal footing at the Planck scale, which is a scenario that could induce minuscule violations of Lorentz invariance. On the one hand, indications for such violations exist in closed-string theories [1-5] and loop quantum gravity [6,7]. On the other hand, models of noncommutative spacetimes [8,9], spacetime foam models [10-12], (chiral) field theories defined on spacetimes with nontrivial topologies [13-16], and Hořava-Lifshitz gravity [17] are based on the assumption that Lorentz invariance is violated. For several decades, violations of this fundamental symmetry have been looked for where until the end of the last millennium, these experimental searches had been rather unsystematic. To be able to compare the results of different experiments to each other and to make theoretical predictions of possible experimental signals, the minimal standard-model extension (SME) was established in $[18,19]$. The minimal SME is a field-theory framework parametrizing all power-counting renormalizable Lorentzviolating contributions that are consistent with both coordinate covariance and the gauge structure of the standard model. Each contribution is decomposed into controlling coefficients and a field operator suitably contracted to form an observer Lorentz scalar. In gravity, Lorentz invariance is a local concept and a parametrization of deviations from local Lorentz invariance, local position invariance, and the

\footnotetext{
jalfieres@gmail.com

marco.schreck@ufma.br
}

Published by the American Physical Society under the terms of the Creative Commons Attribution 4.0 International license. Further distribution of this work must maintain attribution to the author(s) and the published article's title, journal citation, and DOI. Funded by SCOAP ${ }^{3}$. weak equivalence principle in terms of minimal operators was provided in [20]. The nongravitational part of the SME was extended in [21-23] to include all operators of arbitrary mass dimension where this generalization is called the nonminimal SME. It is important to recall that $C P T$ violation implies Lorentz violation in effective field theory in Minkowski spacetime [24], which is why all $C P T$-violating operators are contained in the SME, as well.

Most experimental tests of gravity are performed with classical test bodies, cf. [25] for a compilation of all current constraints on controlling coefficients. General relativity is a classical theory, after all. However, the field-theory description of the SME is not entirely suitable to predict effects of Lorentz violation within classical physics, which is why it would be highly desirable to have a map from the Lagrange density in field theory to the classical Lagrangian of a relativistic, pointlike particle. A map that provides a classical Lagrangian from the field-theory dispersion equation was constructed explicitly in [26]. Based on this construction, the first classical Lagrangians for a wide range of controlling coefficients within the minimal SME were obtained in the same paper. These results are exact and are composed of Lagrangians for a combination of $a, c$, $e, f$ coefficients, the full Lagrangian for the $b$ coefficients, and partial results for the $d, H$ coefficients. This set of Lagrangians was complemented by results for more involved families of $d, g$ coefficients given in $[27,28]$.

Based on some of these findings, the motion of a charged, classical particle under the influence of both a Lorentzviolating background field and an electromagnetic field was studied in [29]. In addition, the modified time evolution of a semiclassical analog of spin was examined with the BMT equation. In [30] it was demonstrated that the procedure of finding Lagrangians from the field theory description can be reversed at first order in Lorentz violation. Thus, it is possible to reobtain parts of the SME Hamilton operator from a 
particular classical Lagrangian by the usual quantization procedure. Finally, an approach was developed in [31] to assign Finsler structures to the minimal SME photon sector. This procedure is based on modified refractive indices and the eikonal equation.

The first exact nonminimal Lagrangian was derived in [32] for the isotropic operator $\hat{m}^{(5)}$. However, the latter is highly complicated, nontransparent and too unwieldy to be used in applications. Therefore, subsequently it was found to be more reasonable to obtain perturbative expansions of such Lagrangians in the nonminimal SME. The method of Gröbner bases, which is a generalization of Gauß' algorithm for nonlinear systems of equations, provides a powerful technique to derive leading-order Lagrangians for the lowest-dimensional contributions to the spindegenerate operators, i.e., for $\hat{a}^{(5)}, \hat{c}^{(6)}, \hat{e}^{(6)}, \hat{f}^{(6)}$, and $\hat{m}^{(5)}$ [33]. These Lagrangians are natural generalizations of the first-order minimal ones with the controlling coefficients replaced by suitable contractions of the nonminimal coefficients and the four-velocity. An additional scalar parameter before the Lorentz-violating contribution ensures that its mass dimension is consistent with that of the standard term and that it is positively homogeneous of degree one in the velocity. The latter is a reasonable property to require, as it leads to an action that is invariant under reparametrizations of the worldline.

It was shown in [34] that such classical Lagrangians can be promoted to Finsler structures by a procedure that has parallels to a Wick rotation. The Lagrangians for the $a, c$, $e$, and $f$ coefficients were found to be related to what is known as a Randers structure, whereas the Lagrangian for $b$ was demonstrated to be linked to a hitherto unknown Finsler space that is not of Randers type. The properties of this Finsler space, which is referred to as $b$ space, were investigated in the latter article. Also, the Finsler structures associated with the coefficients of the nonminimal SME studied in [33] were found to be different from the Randers structure.

Since its discovery, $b$ space has been subject to further studies. Three-dimensional versions of it were demonstrated to play a role in systems of classical mechanics and magnetostatics [35]. Besides that, Finsler $b$ space was discovered to have singularities that can be removed with a desingularization procedure [36] whose existence is based on the famous Hironaka theorem for algebraic varieties. An alternative to this procedure, which is related to the formalism of extended Hamiltonians introduced by Dirac, was presented in the recent work [37]. In principle, $b$ space is a special case of a more general type of Finsler structures that are called bipartite and that are focused on in [38].

The objective of the current paper is to provide a complete generalization of the results of [33] that covers the whole SME at first order in Lorentz violation. Special emphasis is put on the spin-nondegenerate operators $\hat{b}, \hat{d}$, $\hat{H}$, and $\hat{g}$ that were not treated in the previous reference. The paper is organized, as follows. At the beginning of Sec. II, we recall the basics on how to obtain a classical Lagrangian from the corresponding SME field theory. The five equations describing the map from the wave packet to a classical, pointlike particle will be introduced. This section is also dedicated to deriving such Lagrangians for both the spin-degenerate and spin-nondegenerate operators including a subsequent discussion of the results. Based on the Lagrangians obtained to that point, in Sec. III we will be in a position to state the first-order result covering the full SME, which is the central result of the current article. Finally, Sec. IV provides some concluding remarks on all findings. In addition, it will be demonstrated analytically that the five nonlinear equations are fulfilled at leading order by the classical Lagrangians obtained. As these proofs for the spin-nondegenerate operators are lengthy, they can be found in Appendices A and B, respectively. The paper rests on natural units with $\hbar=c=1$.

\section{CONSTRUCTION AND PROPERTIES OF LAGRANGIANS}

We consider the Lagrange density describing nonminimal Lorentz violation in the fermion sector of the SME. Its explicit form is stated in [23]. The map from the fieldtheory description to the classical Lagrange function of a relativistic, pointlike particle of mass $m_{\psi}$ moving with fourvelocity $u^{\mu}$ is governed by the following set of five ordinary nonlinear equations [26]:

$$
\begin{aligned}
\mathcal{R}(p) & =0, \\
\frac{\partial p_{0}}{\partial p_{i}} & =-\frac{u^{i}}{u^{0}}, \quad i \in\{1,2,3\}, \\
L & =-p_{\mu} u^{\mu}, \quad p_{\mu}=-\frac{\partial L}{\partial u^{\mu}},
\end{aligned}
$$

where $p_{\mu}=\mathrm{i} \partial_{\mu}$ is the momentum that appears in the Fourier decomposition of a wave packet into plane waves. Equation (2.1a) depends on the momentum only and it is simply the dispersion equation following from the determinant of the modified Dirac operator. The set of three equations (2.1b) identifies the group velocity of the centroid of a quantum wave packet with the three-velocity $\mathbf{u} / u^{0}$ of the classical particle. The convention is such that the four-momentum components have lower indices whereas the four-velocity components are supposed to have upper ones. Hence, an additional sign must be taken into account on the right-hand side. Furthermore, the classical Lagrange function is supposed to be positively homogeneous in the velocity, which means that $L(\lambda u)=$ $\lambda L(u)$ for $\lambda>0$. This property grants parametrization invariance of the action, which is a must-have in physics, as the action should only depend on the path, but not on the way how it is parametrized. Equation (2.1c) is famous Euler's theorem that follows from exactly the latter 
characteristic of the Lagrangian. The canonical momentum is defined with a minus sign to grant the correct sign for the nonrelativistic kinetic energy.

Solving the five equations for $p^{\mu}=p^{\mu}(u)$ and $L=L(u)$ corresponds to an explicit construction of the map. However, as the system is nonlinear, it is highly nontrivial to solve it-even in the context of the minimal SME. Gröbner bases provide a tool to treat Eqs. (2.1) systematically, which has proven extremely useful to derive Lagrangians in the nonminimal SME [33]. In what follows, classical Lagrangians will be obtained that fulfill Eqs. (2.1) at leading order in Lorentz violation. The complete family of nonminimal coefficients ought to be covered. We will restrict our analysis to particles. Classical Lagrangians corresponding to antiparticle dispersion relations can simply be obtained from the particle Lagrangians via the substitution $m_{\psi} \mapsto-m_{\psi}$, cf. [26,27].

\section{A. Spin-degenerate operators}

Let us start deriving the classical Lagrangians for the spin-degenerate operators $\hat{a}, \hat{c}, \hat{e}, \hat{f}$, and $\hat{m}$. Our approach shall be exemplified by the $a$ coefficients. The minimal $a$ coefficients constitute an observer four-vector and they are contracted with a field operator of mass dimension 3 . In the nonminimal SME, the number of additional derivatives in the field operator subsequently increases by two whereupon the number of indices of the controlling coefficients rises by two and the mass dimension decreases by two. The Lagrangian for $d=3$ was obtained in [26] where for $d=5$ it was found in [33] based on the method of Gröbner bases. We recall both Lagrangians:

$$
\begin{aligned}
L^{a^{(3)}} & =-m_{\psi} \sqrt{u^{2}}-a_{*}^{(3)}, \\
a_{*}^{(3)} & \equiv a_{\mu}^{(3)} u^{\mu}, \\
L^{\hat{a}^{(5)}} & =-m_{\psi} \sqrt{u^{2}}-\frac{m_{\psi}^{2}}{u^{2}} \hat{a}_{*}^{(5)}+\cdots, \\
\hat{a}_{*}^{(5)} & \equiv a_{\mu \nu}^{(5)} u^{\mu} u^{\nu} u^{\varrho} .
\end{aligned}
$$

A hat is put on contractions associated with nonminimal operators. The minimal result is exact and the nonminimal one is valid at first order in Lorentz violation. Neglected higher-order contributions will be indicated as ellipses. ${ }^{1}$ Due to observer Lorentz invariance, consistency of the mass dimension, and positive homogeneity of degree 1 in the velocity, the perturbative form of a Lagrangian is quite restricted. The Lorentz-violating contribution must involve the component coefficients. Suitable observer Lorentz scalars $\hat{a}_{*}^{(d)}$ can be formed by contracting the latter

\footnotetext{
${ }^{1}$ Ellipses used in computations indicate terms discarded after a certain step. This procedure makes it easier for the reader to keep track of leading-order approximations.
}

coefficients with the only four-vector available, which is the four-velocity. ${ }^{2}$ The mass dimension is made consistent with that of the standard term by introducing additional powers of the fermion mass. Positive homogeneity of the second term is restored by suitable powers of the Lorentz scalar $u^{2}$ in the denominator. These general arguments enable us to propose an Ansatz for arbitrary odd $d \geq 3$ :

$$
\begin{aligned}
L^{\hat{a}^{(d)}} & =-m_{\psi} \sqrt{u^{2}}-\hat{A}_{*}+\cdots, \\
\hat{A}_{*} & \equiv \Xi_{a} \hat{a}_{*}, \quad \Xi_{a}=\Xi_{a}^{(d)}=\frac{m_{\psi}^{d-3}}{\left(u^{2}\right)^{(d-3) / 2}}, \\
\hat{a}_{*} & =\hat{a}_{*}^{(d)} \equiv a_{\mu \alpha_{1} \ldots \alpha_{d-3}}^{(d)} u^{\mu} u^{\alpha_{1}} \ldots u^{\alpha_{d-3}},
\end{aligned}
$$

where this result is supposed to be valid at first order in the controlling coefficients. It is convenient to define a second Lorentz scalar $\hat{A}_{*}$ that corresponds to the product of the consistency factor $\Xi_{a}$ and the Lorentz scalar obtained by contracting the controlling coefficients with the four-velocity. Note that $\hat{A}_{*}$ is homogeneous of degree 1 in contrast to $\hat{a}_{*}$, as each of the $d-2$ indices is contracted with a four-velocity to be compensated by the factor $\left(u^{2}\right)^{(d-3) / 2}$ in the denominator. The usefulness of $\hat{A}_{*}$ will become clear below when a general proof of the validity of the Lagrangian at leading order in Lorentz violation will be provided.

It can be checked that for $d=3,5$ the Ansatz reproduces the already known results of Eqs. (2.2). Note that in what follows, the index indicating the mass dimension at various quantities will be dropped to simplify the notation, i.e., it will be mentioned only when necessary. A formal proof of the validity of the Lagrangian is feasible and it is demonstrated as follows, i.e., we will show that the Lagrangian fulfills Eqs. (2.1) at leading order in Lorentz violation. The starting point is the covariant momentum as a function of the fourvelocity, which is obtained from the Lagrangian via

$$
p_{\mu}=-\frac{\partial L^{\hat{a}^{(d)}}}{\partial u^{\mu}}=m_{\psi} \frac{u_{\mu}}{\sqrt{u^{2}}}+\frac{\partial \hat{A}_{*}}{\partial u^{\mu}},
$$

cf. Eq. (2.1c). Note that here it is already much more convenient to express the Lagrangian in terms of $\hat{A}_{*}$, as it is not necessary to include the derivative of $\Xi_{a}$ explicitly. When the Lagrangian is correct, as it stands, this momentum must obey the dispersion equation at first order in Lorentz violation, which is quite simple to show for the $a$ coefficients:

$$
\begin{gathered}
\mathcal{R}=(p-\hat{a})^{2}-m_{\psi}^{2}=p^{2}-2 \hat{a} \cdot p-m_{\psi}^{2}+\cdots, \\
\hat{a}_{\mu}=\hat{a}_{\mu}^{(d)} \equiv a_{\mu \alpha_{1} \ldots \alpha_{d-3}} p^{\alpha_{1}} \ldots p^{\alpha_{d-3}} .
\end{gathered}
$$

\footnotetext{
${ }^{2}$ Throughout the article, an asterisk indicates controlling coefficients suitably contracted with four-velocities. This notation was introduced in [33] and it will be adopted for consistency.
} 
Here, $\hat{a}_{\mu}$ is a four-vector formed from a suitable contraction of the controlling coefficients with the four-momentum. At leading order in Lorentz violation, it suffices to replace each four-momentum by the standard expression $m_{\psi} u_{\mu} / \sqrt{u^{2}}$ producing $\hat{a}_{\mu} \approx \Xi_{a} \hat{a}_{* \mu}$. Inserting the momentum and the latter approximate relationship into the dispersion equation gives

$\mathcal{R}=m_{\psi}^{2}+\frac{2 m_{\psi}}{\sqrt{u^{2}}} u^{\mu} \frac{\partial \hat{A}_{*}}{\partial u^{\mu}}-\frac{2 m_{\psi}}{\sqrt{u^{2}}} \hat{A}_{*}-m_{\psi}^{2}+\cdots=0$.

It is very convenient to employ Euler's theorem, which is applicable, as $\hat{A}_{*}$ is positively homogeneous of degree 1 in the four-velocity:

$$
u^{\mu} \frac{\partial \hat{A}_{*}}{\partial u^{\mu}}=\hat{A}_{*} .
$$

Hence, the dispersion equation is fulfilled when neglecting higher-order contributions in Lorentz violation. The next step is to verify Eq. (2.1b). We do this by computing the first implicit derivative of the dispersion equation with respect to $p_{i}$, replace $\partial p_{0} / \partial p_{i}$ by $-u^{i} / u^{0}$, and insert the canonical momentum of Eq. (2.4):

$$
\begin{aligned}
\frac{\partial \mathcal{R}}{\partial p_{i}}= & 2 p_{0} \frac{\partial p_{0}}{\partial p_{i}}+2 p^{i}-2 \frac{\partial(\hat{a} \cdot p)}{\partial p_{i}}+\cdots \\
= & 2\left[m_{\psi} \frac{u^{0}}{\sqrt{u^{2}}}+\frac{\partial \hat{A}_{*}}{\partial u^{0}}\right]\left(-\frac{u^{i}}{u^{0}}\right)+2\left(m_{\psi} \frac{u^{i}}{\sqrt{u^{2}}}-\frac{\partial \hat{A}_{*}}{\partial u^{i}}\right) \\
& -2 \frac{\partial(\hat{a} \cdot p)}{\partial p_{i}} \\
= & -2\left(\frac{\partial \hat{A}_{*}}{\partial u^{0}} \frac{u^{i}}{u^{0}}+\frac{\partial \hat{A}_{*}}{\partial u^{i}}\right)-2 \frac{\partial(\hat{a} \cdot p)}{\partial p_{i}}=0
\end{aligned}
$$

where the derivative of $\hat{a} \cdot p$ by the momentum was expressed in terms of the derivative of $\hat{A}_{*}$ with respect to the four-velocity components:

$$
\begin{aligned}
\frac{\partial(\hat{a} \cdot p)}{\partial p_{i}} & =m_{\psi} \frac{\partial\left(\Xi_{a} \hat{a}_{* \mu} u^{\mu} / \sqrt{u^{2}}\right)}{\partial u^{\sigma}} \frac{\partial u^{\sigma}}{\partial p_{i}}+\cdots \\
& =m_{\psi}\left(\frac{\partial\left(\hat{A}_{*} / \sqrt{u^{2}}\right)}{\partial u^{0}} \frac{\partial u^{0}}{\partial p_{i}}+\frac{\partial\left(\hat{A}_{*} / \sqrt{u^{2}}\right)}{\partial u^{j}} \frac{\partial u^{j}}{\partial p_{i}}\right) \\
& =-\left(\frac{\partial \hat{A}_{*}}{\partial u^{0}} \frac{u^{i}}{u^{0}}+\frac{\partial \hat{A}_{*}}{\partial u^{i}}\right) .
\end{aligned}
$$

To do so, the zeroth-order correspondence between fourvelocity and four-momentum and the related derivatives are needed

$$
\frac{u^{\mu}}{\sqrt{u^{2}}}=\frac{p^{\mu}}{\sqrt{p^{2}}}=\frac{p^{\mu}}{m_{\psi}}
$$

$\frac{1}{\sqrt{u^{2}}} \frac{\partial u^{0}}{\partial p_{i}}=\frac{1}{m_{\psi}} \frac{p_{i}}{p^{0}}=-\frac{1}{m_{\psi}} \frac{u^{i}}{u^{0}}, \quad \frac{1}{\sqrt{u^{2}}} \frac{\partial u^{j}}{\partial p_{i}}=-\frac{1}{m_{\psi}} \delta^{i j}$,

in combination with the following property for a generic function $f=f\left(u^{2}\right)$ that depends on the four-velocity squared only:

$\frac{u^{i}}{u^{0}} \frac{\partial f\left(u^{2}\right)}{\partial u^{0}}+\frac{\partial f\left(u^{2}\right)}{\partial u^{i}}=\frac{u^{i}}{u^{0}} \frac{\partial f}{\partial\left(u^{2}\right)} 2 u^{0}-\frac{\partial f}{\partial\left(u^{2}\right)} 2 u^{i}=0$.

Thus, the term involving the derivative of $1 / \sqrt{u^{2}}$ vanishes in Eq. (2.9) whereupon the remaining result compensates the first term in Eq. (2.8). As the Lagrangian is positively homogeneous of degree 1 in the velocity, Eq. (2.1c) is fulfilled automatically. Note that Eq. (2.10b) is valid for a parametrization with constant $u^{2}$. Due to parametrization invariance of the setting, the result should also be valid for an arbitrary parametrization.

To summarize, in contrast to the derivations of classical Lagrangians in [33], which relied on the method of Gröbner bases, the current Lagrangian was simply obtained by making a suitable guess that is in accordance with observer Lorentz invariance and positive homogeneity of first degree in the velocity. Furthermore, it ought to reproduce the already known results. The same procedure is now successfully employed to arrive at the classical Lagrangians for the remaining spin-degenerate operators. The general result for the classical Lagrangian at leading order in Lorentz violation is reasonably expressed in the form

$$
L^{\hat{x}^{(d)}}=-m_{\psi} \sqrt{u^{2}}-\hat{X}_{*}+\cdots, \quad \hat{X}_{*}=\Xi_{x} \hat{x}_{*} .
$$

Here $\hat{x}_{*}=\hat{x}_{*}^{(d)}$ is a total contraction of controlling coefficients with an appropriate combination of four-velocities. The parameter $\Xi_{x}=\Xi_{x}^{(d)}$ only involves the particle mass and a suitable power of the observer scalar $u^{2}$. In the general case, it is also convenient to define observer scalars $\hat{X}_{*}=\hat{X}_{*}^{(d)}$ that are positively homogeneous of degree 1 in the velocity. The explicit expressions of these quantities for the complete set of spin-degenerate operators are listed in Table I.

Several remarks on the classical Lagrangian of Eq. (2.12) are in order. First, the Lagrangian is a sum of the standard term $L=-m_{\psi} \sqrt{u^{2}}$ and a contribution that is linear in the controlling coefficients. Second, it is formed from observer Lorentz scalars to render it a Lorentz scalar, as expected. ${ }^{3}$

\footnotetext{
${ }^{3}$ Parametrizing the world line of the particle with proper time $\tau$, the four-velocity is defined by $u^{\mu}=\gamma(1, \mathbf{v})^{\mu}$, as usual. The latter is a four-vector that satisfies $u^{2}=1$. In this parametrization, a point-particle Lagrangian is a Lorentz scalar. Due to parametrization invariance of the action, different parametrizations with a suitably chosen $\lambda$ may be useful [26]. In such a case, $u^{2}=$ $(\mathrm{d} \tau / \mathrm{d} \lambda)^{2}$, i.e., $u^{\mu}$ is not a four-vector anymore, rendering the Lagrangian noninvariant under Lorentz transformations.
} 
TABLE I. Parameters of the generic classical Lagrangian of Eq. (2.12). The first column states the observer scalar employed in the Lagrangian where the entries in the second column give the corresponding explicit expressions. The consistency factors are listed in the third column. The fourth column shows Euler's theorem for each of the Lorentz scalars defined in the first two columns. The fifth column states the leading-order results for the Lorentz-violating operators in momentum space with each four-momentum expressed in terms of the four-velocity.

\begin{tabular}{lcccc}
\hline \hline$\hat{x}_{*}$ & Explicit contraction & $\Xi_{x}$ & Radial derivative & Correspondence \\
\hline$\hat{a}_{*}$ & $a_{\mu \alpha_{1} \ldots \alpha_{d-3}}^{(d)} u^{\mu} u^{\alpha_{1}} \ldots u^{\alpha_{d-3}}$ & $m_{\psi}^{d-3} /\left(u^{2}\right)^{(d-3) / 2}$ & $u^{\mu} \frac{\partial \hat{a}_{*}}{\partial u^{\mu}}=(d-2) \hat{a}_{*}$ & $\hat{a}_{\mu} \approx \Xi_{a} \hat{a}_{* \mu}$ \\
$\hat{c}_{*}$ & $c_{\mu \alpha_{1} \ldots \alpha_{d-3}}^{(d)} u^{\mu} u^{\alpha_{1}} \ldots u^{\alpha_{d-3}}$ & $-m_{\psi}^{d-3} /\left(u^{2}\right)^{(d-3) / 2}$ & $u^{\mu} \frac{\partial \hat{c}_{*}}{\partial u^{\mu}}=(d-2) \hat{c}_{*}$ & $\hat{c}_{\mu} \approx-\Xi_{c} \hat{c}_{* \mu}$ \\
$\hat{e}_{*}$ & $e_{\alpha_{1} \ldots \alpha_{d-3}}^{(d)} u^{\alpha_{1}} \ldots u^{\alpha_{d-3}}$ & $-m_{\psi}^{d-3} /\left(u^{2}\right)^{(d-4) / 2}$ & $u^{\mu} \frac{\partial \hat{e}_{*}}{\partial u^{\mu}}=(d-3) \hat{e}_{*}$ & $\hat{e}_{\mu} \approx-\frac{\Xi_{e}}{m_{\psi}} \hat{e}_{* \mu}$ \\
$\hat{f}_{*}$ & $f_{\alpha_{1} \ldots \alpha_{d-3}}^{(d)} u^{\alpha_{1}} \ldots u^{\alpha_{d-3}}$ & $m_{\psi}^{2 d-7} /\left[2\left(u^{2}\right)^{(2 d-7) / 2}\right]$ & $u^{\mu} \frac{\partial \hat{f}_{*}^{2}}{\partial u^{\mu}}=2(d-3) \hat{f}_{*}^{2}$ & $\hat{f}_{\mu} \hat{f}_{\nu} \approx \frac{2 \sqrt{u^{2} \Xi_{f}}}{m_{\psi}} \hat{f}_{* \mu} \hat{f}_{* \nu}$ \\
$\hat{m}_{*}$ & $m_{\alpha_{1} \ldots \alpha_{d-3}}^{(d)} u^{\alpha_{1}} \ldots u^{\alpha_{d-3}}$ & $m_{\psi}^{d-3} /\left(u^{2}\right)^{(d-4) / 2}$ & $u^{\mu} \frac{\partial \hat{m}_{*}}{\partial u^{\mu}}=(d-3) \hat{m}_{*}$ & $\hat{m} \approx \frac{\Xi_{m}}{\sqrt{u^{2}}} \hat{m}_{*}$ \\
$\hat{\mathcal{S}}_{*}$ & $\mathcal{S}_{\alpha_{1} \ldots \alpha_{d-3}}^{(d)} u^{\alpha_{1}} \ldots u^{\alpha_{d-3}}$ & $-m_{\psi}^{d-3} /\left(u^{2}\right)^{(d-4) / 2}$ & $u^{\mu} \frac{\partial_{*}}{\partial u^{\mu}}=(d-3) \hat{\mathcal{S}}_{*}$ & $\hat{\mathcal{S}} \approx-\frac{\Xi_{\mathcal{S}}}{\sqrt{u^{2}}} \hat{\mathcal{S}}_{*}$ \\
$\hat{\mathcal{V}}_{*}$ & $\mathcal{V}_{\mu \alpha_{1} \ldots \alpha_{d-3}}^{(d)} u^{\mu} u^{\alpha_{1}} \ldots u^{\alpha_{d-3}}$ & $-m_{\psi}^{d-3} /\left(u^{2}\right)^{(d-3) / 2}$ & $u^{\mu} \frac{\hat{\mathcal{V}}_{*}}{\partial u^{\mu}}=(d-2) \hat{\mathcal{V}}_{*}$ & $\hat{\mathcal{V}}_{\mu} \approx-\Xi_{\mathcal{V}} \hat{\mathcal{V}}_{* \mu}$ \\
\hline \hline
\end{tabular}

Third, it has to be of mass dimension 1 where additional powers of masses must be introduced in the Lorentzviolating term such that its mass dimension corresponds to the mass dimension of the standard term. Fourth, additional powers of the four-velocity squared are needed to make the nonstandard contribution homogeneous of degree 1 in the velocity. Fifth, there is the correspondence $\hat{a}_{*} \leftrightarrow-m_{\psi} \hat{e}_{*}$, which is the generalization of $a_{\mu} \leftrightarrow-m_{\psi} e_{\mu}$ found for the minimal SME [28]. Sixth, the dimensionless number $\left|\hat{X}_{*}\right| /\left(m_{\psi} \sqrt{u^{2}}\right)$ must be $\ll 1$ such that the first-order approximation is justified. This requirement translates into the additional condition that $\mathbf{u}^{2}$ should not lie in the close vicinity of $u_{0}^{2}$. When we use a parametrization of the particle trajectory such that $u^{0}=1$ and $\mathbf{u}=\mathbf{v}$ with the three-velocity $\mathbf{v}$, this condition means that the particle should not travel with a velocity too close to the speed of light. The condition $\left|\hat{X}_{*}\right| /\left(m_{\psi} \sqrt{u^{2}}\right) \ll 1$ can be solved for one of the spatial velocity components on a case-by-case basis. As an example, let us consider an observer frame with the only nonzero coefficient $a_{000}^{(5)}$. As this case is isotropic, the inequality can even be solved for $|\mathbf{u}|$ leading to

$$
|\mathbf{u}| \ll \sqrt{u_{0}^{2}-\left(m_{\psi}\left|u_{0}^{3} a_{000}^{(5)}\right|\right)^{2 / 3}} .
$$

Inserting the numbers $u_{0}=1$ and $m_{\psi} a_{000}^{(5)}=10^{-10}$ produces $|\mathbf{u}| \ll 1-10^{-7}$, i.e., the condition is already fulfilled for a $\beta$ factor of the particle that amounts to around $1-10^{-6}$. This result demonstrates that the perturbation can be much smaller than the standard term even for ultrarelativistic particles. It is not a requirement that the particle moves nonrelativistically. The analog statement in momentum space is that the energy and momentum must be small enough, as the relevance of a nonminimal contribution rises with increasing momentum.

For the remaining spin-degenerate operators, the proof that Eq. (2.12) fulfills Eqs. (2.1) works completely analogously when the corresponding expressions of Table I are employed. Furthermore, it is now quite convenient to generalize the Lagrangian of Eq. (2.12) to the situation when operators of different mass dimensions are added, e.g., $\hat{a}_{\mu} \mapsto \sum_{d \geq 3} a_{\mu \alpha_{1} \ldots \alpha_{d-3}}^{(d)} p^{\alpha_{1}} \ldots p^{\alpha_{d-3}}$ for the $a$ coefficients. We then simply have to replace the above observer scalar $\hat{X}_{*}^{(d)}$ for a particular mass dimension $d$ by a sum, i.e., $\hat{X}_{*}^{(d)} \mapsto \sum_{d} \hat{X}_{*}^{(d)}$ where $d$ runs over suitable values permitted for the operator $\hat{x}^{(d)}$. As each individual summand of the introduced sum is positively homogeneous of degree 1 in the velocity, the sum itself will have that property, too. Hence, the proof of the validity of the Lagrangian can be taken over entirely. Finally, the expansion of Lorentz-violating operators in terms of momenta leads to the additional requirement $\hat{X}_{*}^{(d+2)} \ll \hat{X}_{*}^{(d)}$ such that each contribution is suppressed compared to the previous one.

\section{B. Spin-nondegenerate operators}

The base for obtaining classical Lagrangians for the spin-degenerate operators was laid in [33], whereas spinnondegenerate operators were not considered in the latter paper. Hence, not a single classical Lagrangian has been derived for the nonminimal operators $\hat{b}, \hat{d}, \hat{H}$, and $\hat{g}$ until now. However, at least a couple of minimal results are known such as that for the $b$ coefficients, cf. Eq. (12) of [26] for $a^{(3) \mu}=0$ :

$$
L^{b^{(3)}}=-m_{\psi} \sqrt{u^{2}} \mp \sqrt{\left(b^{(3)} \cdot u\right)^{2}-\left(b^{(3)}\right)^{2} u^{2}},
$$

where there are two distinct Lagrangians for particles because of spin-nondegeneracy. When we assume that the previously used technique also works for the spinnondegenerate operators, we could propose a proper Ansatz that is in accordance with observer Lorentz invariance 
and positive homogeneity of degree 1 in the velocity. Furthermore, it should reproduce the minimal result $L^{b^{(3)}}$ :

$$
\begin{aligned}
L^{\hat{b}^{(d)}} & =-m_{\psi} \sqrt{u^{2}} \mp \hat{\mathcal{B}}_{*}+\cdots, \\
\hat{\mathcal{B}}_{*} & =\sqrt{\hat{B}_{*}^{2}-\left(\hat{B}_{*}^{\mu} \hat{B}_{* \mu}\right) u^{2},} \\
\hat{B}_{* \mu} & \equiv \Xi_{b} \hat{b}_{* \mu}, \quad \hat{B}_{*} \equiv \Xi_{b} \hat{b}_{*}, \\
\Xi_{b} & =\Xi_{b}^{(d)}=\frac{m_{\psi}^{d-3}}{\left(u^{2}\right)^{(d-3) / 2}}, \\
\hat{b}_{* \mu} & =\hat{b}_{* \mu}^{(d)} \equiv b_{\mu \alpha_{1} \ldots \alpha_{d-3}}^{(d)} u^{\alpha_{1}} \ldots u^{\alpha_{d-3}}, \\
\hat{b}_{*} & =\hat{b}_{*}^{(d)} \equiv \hat{b}_{* \mu} u^{\mu} .
\end{aligned}
$$

An analytical proof of the validity of the Lagrangian for arbitrary odd $d \geq 3$ is tedious but feasible. Readers who are only interested in the results can skip the proof, which is why it has been moved to Appendix A. In this context it is crucial to recall that at the level of the dispersion equation, the $b$ and $d$ coefficients contribute to the pseudovector operator $\hat{\mathcal{A}}^{\mu}$. Hence, the dispersion equation for $\hat{d}^{\mu \nu}$ at leading order in Lorentz violation follows from the dispersion equation of $\hat{b}^{\mu}$ in replacing the latter by $-\hat{d}^{\mu \nu} \bar{p}_{\nu}$ where $\bar{p}_{\mu} \equiv\left(E_{0}, \mathbf{p}\right)_{\mu}$ with the standard dispersion relation $E_{0}=\left(\mathbf{p}^{2}+m_{\psi}^{2}\right)^{1 / 2}$. The correspondence at the level of classical Lagrangians is then simply $\hat{b}_{* \mu} \leftrightarrow-\hat{d}_{* \mu \nu} u^{\nu}=-\hat{d}_{* \mu}$ at first order in Lorentz violation. The analytical proof for the $b$ coefficients can literally be taken over to the $d$ coefficients where the only difference is that the mass dimension takes even values $\geq 4$.

A similar procedure is applied to the $\hat{H}$ and $\hat{g}$ operators. Let $X \equiv H_{\mu \nu}^{(3)} H^{(3) \mu \nu} / 4$ and $Y \equiv H_{\mu \nu}^{(3)} \tilde{H}^{(3) \mu \nu} / 4$ with $\tilde{H}^{(3) \mu \nu}$ corresponding to the dual of $H^{(3) \mu \nu}$. The exact classical Lagrangian of the minimal $H$ coefficients for the configuration characterized by $Y=0$ is given by Eq. (15) in [26] and it reads

$$
\begin{aligned}
\left.L^{H^{(3)}}\right|_{Y=0} & =-m_{\psi} \sqrt{u^{2}} \mp \sqrt{u^{\nu}\left(H^{(3)}\right)^{\mu}{ }_{\nu} H_{\mu \varrho}^{(3)} u^{\varrho}+2 X u^{2}} \\
& =-m_{\psi} \sqrt{u^{2}} \mp \sqrt{-\left(\tilde{H}^{(3)}\right)^{\mu}{ }_{\nu} u^{\nu} \tilde{H}_{\mu \varrho}^{(3)} u^{\varrho}},
\end{aligned}
$$

where we used

$$
\left(H^{(3)}\right)^{\mu}{ }_{\nu} u^{\nu} H_{\mu \varrho}^{(3)} u^{\varrho}=\left(\tilde{H}^{(3)}\right)^{\mu}{ }_{\nu} u^{\nu} \tilde{H}_{\mu \varrho}^{(3)} u^{\varrho}+2 X u^{2} .
$$

Note that this Lagrangian is exact in Lorentz violation. Based on the same fundamental principles as before, we can propose a suitable Ansatz to cover the $H$ coefficients for arbitrary mass dimension at first order in Lorentz violation:

$$
L^{\hat{H}^{(d)}}=-m_{\psi} \sqrt{u^{2}} \mp \hat{\mathcal{H}}_{*}+\cdots, \quad \hat{\mathcal{H}}_{*}=\sqrt{-\tilde{\hat{H}}_{*}^{\mu} \tilde{\hat{H}}_{* \mu}},
$$

$$
\begin{aligned}
& \tilde{\hat{H}}_{* \mu} \equiv \Xi_{h} \tilde{\hat{h}}_{* \mu}, \quad \Xi_{H}=\Xi_{H}^{(d)}=\frac{m_{\psi}^{d-3}}{\left(u^{2}\right)^{(d-3) / 2}}, \\
& \tilde{\hat{h}}_{* \mu}=\tilde{\hat{h}}_{* \mu}^{(d)} \equiv \tilde{H}_{\mu \nu \alpha_{1} \ldots \alpha_{d-3}}^{(d)} u^{\nu} u^{\alpha_{1}} \ldots u^{\alpha_{d-3}} .
\end{aligned}
$$

The latter reproduces the minimal result. In Appendix B, the Lagrangian proposed is shown to obey Eqs. (2.1) at first order in Lorentz violation. It is valid for all configurations of $H$ coefficients, as a restriction $Y=0$ generalized to $H_{\mu \nu}^{(d)}$ is not used in the proof. When taking into account that both the $H$ and the $g$ coefficients contribute to the two-tensor operator $\hat{\mathcal{T}}^{\mu \nu}$ in the modified Dirac Lagrange density, the leading-order dispersion equation for $\hat{g}^{\text {uve }}$ follows from that of $\hat{H}^{\mu \nu}$ in replacing $\hat{H}^{\mu \nu}$ by $-\hat{g}^{\mu \nu \rho} \bar{p}_{Q^{\prime}}$. The proof of the validity of the classical Lagrangian can then be adapted by considering $\hat{H}_{* \mu \nu} \leftrightarrow-\hat{g}_{* \mu \nu \varrho} u^{\varrho}=-\hat{g}_{* \mu \nu}$ at first order in the controlling coefficients. Note that the mass dimension is even and $\geq 4$ for $\hat{g}$.

Based on these findings, the general Lagrangian for a spin-nondegenerate operator at leading order is expressed as follows:

$$
L^{\hat{x}^{(d)}}=-m_{\psi} \sqrt{u^{2}} \mp \hat{\mathcal{X}}_{*},
$$

with the quantities listed in Table II. Several remarks are in order. First, the standard Lagrangian is reproduced for vanishing controlling coefficients, as expected. Second, each Lorentz-violating coefficient is multiplied by a parameter $\Xi_{x}$ that only depends on the particle mass, the four-velocity squared, and the mass dimension. This parameter has the same form for each type of spin-nondegenerate operator. However, note that the mass dimensions of the coefficients can differ from each other. Third, Lorentz violation is encoded in a square root of quadratic combinations of coefficients, i.e., the correction is of first order in Lorentz violation. Due to the square root dependence, the Lagrangian is not differentiable in the limit of zero Lorentz violation, though. Fourth, there are two Lagrangians for particles that mirror the two distinct modified dispersion relations present for spin-nondegenerate operators. Fifth, taking a closer look at the Lagrangians reveals the correspondence $\tilde{\hat{H}}_{* \mu} \leftrightarrow m_{\psi} \hat{d}_{* \mu}$ for a $\hat{d}_{\mu \nu}$ that is antisymmetric in the first two indices (cf. [28] for the analog in the minimal SME). Sixth, at first order in Lorentz violation, the Lagrangians for $\hat{b}, \hat{d}, \hat{H}$, and $\hat{g}$ are all of the following form:

$$
L^{\hat{x}^{(d)}}=-m_{\psi} \sqrt{u^{2}} \mp \sqrt{u^{\mu} s_{\mu \nu}^{\hat{x}} u^{\nu}},
$$

where $s_{\mu \nu}^{\hat{x}}=s_{\mu \nu}^{\hat{x}}(u)$ are $4 \times 4$ matrices that are listed in Table IIb explicitly. In principle, Eq. (2.20) can be interpreted 
TABLE II. Ingredients necessary to formulate the classical Lagrangian of Eq. (2.19) for a specific spinnondegenerate operator. The first column of (a) states the basic observer scalars and vectors with their explicit construction given in the second column. The third column lists the consistency factors and the fourth gives Euler's theorem for the quantities defined before. In the fifth column, the reader can find the leading-order correspondences between the Lorentz-violating operators transformed to momentum space and the related parameters with each fourmomentum expressed in terms of the four-velocity. The first column of (b) specifies the functions $\hat{\mathcal{X}}_{*}$ that make up the Lorentz-violating contribution of the Lagrangians. In the second column, the observer scalars and vectors necessary to construct these functions can be found. The third column points out Euler's theorem for each $\hat{\mathcal{X}}_{*}$ and in the fourth column the explicit matrices of the generalized bipartite Lagrangian of Eq. (2.20) are listed.

(a)

\begin{tabular}{llllc}
\hline$\hat{x}_{*}, \hat{x}_{* \mu}$ & Explicit contraction & $\Xi_{x}$ & Radial derivative & Correspondence \\
\hline$\hat{b}_{*}$ & $b_{\mu \alpha_{1} \ldots \alpha_{d-3}}^{(d)} u^{\mu} u^{\alpha_{1}} \ldots u^{\alpha_{d-3}}$ & $m_{\psi}^{d-3} /\left(u^{2}\right)^{(d-3) / 2}$ & $u^{\mu} \frac{\partial \hat{b}_{*}}{\partial u^{\mu}}=(d-2) \hat{b}_{*}$ & $\hat{b}_{\mu} \approx \Xi_{b} \hat{b}_{* \mu}$ \\
$\hat{d}_{*}$ & $d_{\mu \alpha_{1} \ldots \alpha_{d-3}}^{(d)} u^{\mu} u^{\alpha_{1}} \ldots u^{\alpha_{d-3}}$ & $m_{\psi}^{d-3} /\left(u^{2}\right)^{(d-3) / 2}$ & $u^{\mu} \frac{\partial \hat{d}_{*}}{\partial u^{\mu}}=(d-2) \hat{d}_{*}$ & $\hat{d}_{\mu} \approx \Xi_{d} \hat{d}_{* \mu}$ \\
$\tilde{\hat{h}}_{* \mu}$ & $\tilde{H}_{\mu \nu \alpha_{1} \ldots \alpha_{d-3}}^{(d)} u^{\nu} u^{\alpha_{1}} \ldots u^{\alpha_{d-3}}$ & $m_{\psi}^{d-3} /\left(u^{2}\right)^{(d-3) / 2}$ & $u^{\mu} \frac{\partial \hat{\hat{h}}_{* \nu}}{\partial u^{\mu}}=(d-2) \tilde{\hat{h}}_{* \nu}$ & $\tilde{\hat{H}}_{\mu \nu} \approx \Xi_{H} \tilde{\hat{H}}_{* \mu \nu}$ \\
$\tilde{\hat{g}}_{* \mu}$ & $\tilde{g}_{\mu \nu \alpha_{1} \ldots \alpha_{d-3}}^{(d)} u^{\nu} u^{\alpha_{1}} \ldots u^{\alpha_{d-3}}$ & $m_{\psi}^{d-3} /\left(u^{2}\right)^{(d-3) / 2}$ & $u^{\mu} \frac{\partial \tilde{\hat{g}}_{* \nu}}{\partial u^{\mu}}=(d-2) \tilde{\hat{g}}_{* \nu}$ & $\tilde{\hat{g}}_{\mu \nu} \approx \Xi_{g} \tilde{\hat{g}}_{* \mu \nu}$ \\
\hline \hline
\end{tabular}

(b)

\begin{tabular}{lcccc}
\hline$\hat{\mathcal{X}}_{*}$ & Explicit expression & $\hat{X}_{*}$ & Radial derivative & Generalized bipartite matrix $s_{\mu \nu}^{\hat{x}}$ \\
\hline$\hat{\mathcal{B}}_{*}$ & $\sqrt{\hat{B}_{*}^{2}-\left(\hat{B}_{*}^{\mu} \hat{B}_{* \mu}\right) u^{2}}$ & $\hat{B}_{*}=\Xi_{b} b_{*}$ & $u^{\mu} \frac{\partial \hat{\mathcal{B}}_{*}}{\partial u^{\mu}}=\hat{\mathcal{B}}_{*}$ & $\hat{B}_{* \mu} \hat{B}_{* \nu}-\left(\hat{B}_{*}^{Q} \hat{B}_{* \ell}\right) \eta_{\mu \nu}$ \\
$\hat{\mathcal{D}}_{*}$ & $\sqrt{\hat{D}_{*}^{2}-\left(\hat{D}_{*}^{\mu} \hat{D}_{* \mu}\right) u^{2}}$ & $\hat{D}_{*}=\Xi_{d} d_{*}$ & $u^{\mu} \frac{\partial \hat{\mathcal{D}}_{*}}{\partial u^{*}}=\hat{\mathcal{D}}_{*}$ & $\hat{D}_{* \mu} \hat{D}_{* \nu}-\left(\hat{D}_{*}^{Q} \hat{D}_{* \ell}\right) \eta_{\mu \nu}$ \\
$\hat{\mathcal{H}}_{*}$ & $\sqrt{-\tilde{\hat{H}}_{*}^{\mu} \tilde{\hat{H}}_{* \mu}}$ & $\tilde{\hat{H}}_{* \mu}=\Xi_{H} \tilde{\hat{H}}_{* \mu}$ & $u^{\mu} \frac{\partial \hat{\mathcal{H}}_{*}}{\partial u^{\mu}}=\hat{\mathcal{H}}_{*}$ & $-\tilde{\hat{H}}_{*}^{Q} \tilde{\hat{H}}_{* \ell} \eta_{\mu \nu} / u^{2}$ \\
$\hat{\mathcal{G}}_{*}$ & $\sqrt{-\tilde{\hat{G}}_{*}^{\mu} \tilde{\hat{G}}_{* \mu}}$ & $\tilde{\hat{G}}_{* \mu}=\Xi_{g} \tilde{\hat{g}}_{* \mu}$ & $u^{\mu} \frac{\partial \hat{\mathcal{G}}_{*}}{\partial u^{\mu}}=\hat{\mathcal{G}}_{*}$ & $-\tilde{\hat{G}}_{*}^{Q} \tilde{\hat{G}}_{* Q} \eta_{\mu \nu} / u^{2}$ \\
\hline \hline
\end{tabular}

as a generalization of what is known as the bipartite structure within the minimal SME [38]. However, in contrast to its original definition, the matrix $s_{\mu \nu}^{\hat{x}}$ now depends on the fourvelocity explicitly. Note the formal similarities of these matrices for all types of spin-nondegenerate operators when taking into account that $\tilde{\hat{H}}_{*}^{\mu} u_{\mu}=\tilde{\hat{\hat{g}}}_{*}^{\mu} u_{\mu}=0$ because of the antisymmetry of $\tilde{\hat{H}}$ and $\tilde{\hat{g}}$. Seventh, the Lagrangians for the first two types and the latter two types of operators can be combined resulting in Lagrangians expressed in terms of an observer pseudovector and a two-tensor that are defined in analogy to the pseudovector operator $\hat{\mathcal{A}}^{\mu}$ and the dual tensor operator $\tilde{\hat{\mathcal{T}}}^{\mu \nu}$ :

$$
\begin{aligned}
& \left.L^{\hat{\mathcal{A}}^{(d)}}\right|_{\text {odd }}=-m_{\psi} \sqrt{u^{2}} \mp \sqrt{\hat{\mathcal{A}}_{*}^{2}-\left(\hat{\mathcal{A}}_{*}^{\mu} \hat{\mathcal{A}}_{* \mu}\right) u^{2}},
\end{aligned}
$$

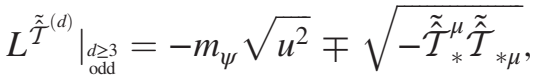

$$
\begin{aligned}
& \hat{\mathcal{A}}_{* \mu}^{(d)} \equiv \Xi_{b}^{(d)}\left(\frac{m_{\psi}}{\sqrt{u^{2}}} \hat{d}_{* \mu}^{(d+1)}-\hat{b}_{* \mu}^{(d)}\right), \\
& \tilde{\hat{\mathcal{T}}}_{* \mu}^{(d)} \equiv \Xi_{H}^{(d)}\left(\frac{m_{\psi}}{\sqrt{u^{2}}} \tilde{\hat{g}}_{* \mu}^{(d+1)}-\tilde{\hat{H}}_{* \mu}^{(d)}\right) .
\end{aligned}
$$

Here, the index indicating the mass dimension of $\hat{\mathcal{A}}_{* \mu}, \tilde{\hat{\mathcal{T}}}_{* \mu}$ is again omitted within the Lagrangians to simplify the notation. The Lagrangians for $\hat{b}, \hat{d}$ follow from Eq. (2.21a) and those for $\hat{H}, \hat{g}$ follow from Eq. (2.21b) for appropriate choices of the coefficients, as expected. These new Lagrangians are of bipartite form, as well.

\section{Map between vector $c$ and pseudoscalar $f$ coefficients}

It is well-known that the minimal $f$ coefficients can be mapped onto the $c$ coefficients by a spinor redefinition [39]. The structure of the map is such that it involves only bilinear combinations of $f$ coefficients and at leading order, it is given by $c_{\mu \nu}^{(4)} \approx-f_{\mu}^{(4)} f_{\nu}^{(4)} / 2$. Comparing the Lagrangians for the $c$ and $f$ coefficients with each other, reveals the following correspondence at leading order:

$$
\begin{gathered}
c_{*}^{(d)} \leftrightarrow-\frac{m_{\psi}^{d-4}}{2\left(u^{2}\right)^{(d-4) / 2}}\left(f_{*}^{(d)}\right)^{2}, \\
c_{* \mu \nu}^{(d)} \leftrightarrow-\frac{m_{\psi}^{d-4}}{2\left(u^{2}\right)^{(d-4) / 2}} f_{* \mu}^{(d)} f_{* \nu}^{(d)} .
\end{gathered}
$$


In the second correspondence, the tensor structure on both sides has been extracted. At leading order, the part of the $c$ coefficients that is symmetric in the first two indices contributes to the Lagrangian only. The known map within the minimal SME is reproduced for $d=4$. However, note that Eqs. (2.22) do not provide a direct map between the controlling coefficients, but just between certain contractions of the $c$ and $f$ coefficients with the four-velocity. Indeed, there exists the alternative possibility of mapping controlling coefficients directly to each other according to the following rule:

$$
c_{\mu \nu \alpha_{2} \ldots \alpha_{d-3}}^{(d)} \leftrightarrow-\frac{1}{2} f_{\mu \alpha_{2} \ldots \alpha_{d / 2-1}}^{(d / 2+2)} f_{\nu \alpha_{d / 2+1} \ldots \alpha_{d-2}}^{(d / 2+2)} .
$$

It can be deduced immediately that this generalization contains the correspondence within the minimal SME. Furthermore, counting the number of indices on each side produces $d-2$, i.e., the map is consistent in this respect. We obtain the Lagrangian for $\hat{f}^{(d)}$ by inserting the latter map into the Lagrangian for $\hat{c}^{(d)}$ and adapting the mass dimension appropriately:

$$
\begin{aligned}
L^{\hat{c}^{(d)}} & =-m_{\psi} \sqrt{u^{2}}-\Xi_{c}^{(d)} \hat{c}_{*}^{(d)} \leftrightarrow-m_{\psi} \sqrt{u^{2}}+\frac{\Xi_{c}^{(d)}}{2}\left(\hat{f}_{*}^{(d / 2+2)}\right)^{2} \\
& =-m_{\psi} \sqrt{u^{2}}+\frac{\Xi_{c}^{\left(2 d^{\prime}-4\right)}}{2}\left(\hat{f}_{*}^{\left(d^{\prime}\right)}\right)^{2} \\
& =-m_{\psi} \sqrt{u^{2}}+\Xi_{f}^{\left(d^{\prime}\right)}\left(\hat{f}_{*}^{\left(d^{\prime}\right)}\right)^{2}=L^{\hat{f}^{\left(d^{\prime}\right)}} .
\end{aligned}
$$

There is one caveat, though. In principle, $d=4+2 n$ with $n \in \mathbb{N}_{0}$ can be chosen for the $c$ coefficients, which directly produces a product of two $f_{\mu \alpha_{2} \ldots \alpha_{n+1}}^{(4+n)}$ on the right-hand side of Eq. (2.23). This counting would permit arbitrary mass dimensions for $f$ as long as they are $\geq 4$. In analogy to the $c$ coefficients, the mass dimension of the $f$ coefficients only takes values $d=4+2 n$, i.e., there is a contradiction. The latter is resolved when restricting the map to $c_{\mu \nu \alpha_{2} \ldots \alpha_{4 n+1}}^{(4 n+4)}$. For $n=1$ this means that $f_{\mu \alpha_{1} \alpha_{2}}^{(6)}$ can only be mapped to $c_{\mu \nu \alpha_{2} \ldots \alpha_{5}}^{(8)}$ where the case $n=2$ describes a mapping between $f_{\mu \alpha_{1} \ldots \alpha_{4}}^{(8)}$ and $c_{\mu \nu \alpha_{2} \ldots \alpha_{9}}^{(12)}$, etc. Therefore, only a subset of $c$ coefficients has a direct connection to $f$ coefficients, whereas the $c_{\mu \nu \alpha_{2} \alpha_{3}}^{(6)}, c_{\mu \nu \alpha_{2} \ldots \alpha_{7}}^{(10)}$, etc. do not have an $f$ counterpart.

\section{Effective coefficients}

At first order in Lorentz violation, suitable field redefinitions allow for combining controlling coefficients of different mass dimension such that new coefficients can be defined that are known as effective, cf. Eqs. (27) of [23]. Based on this observation, it is possible to define effective observer scalars $\hat{a}_{*, \text { eff }}, \hat{c}_{*, \text { eff }}$. Generically,

$$
\begin{aligned}
\hat{X}_{*, \mathrm{eff}}^{(d)} & \equiv \Xi_{x}^{(d)} \hat{x}_{*, \mathrm{eff}}^{(d)}, \\
\hat{x}_{*, \mathrm{eff}}^{(d)} & \equiv x_{\mathrm{eff}, \mu \alpha_{1} \ldots \alpha_{d-3}}^{(d)} u^{\mu} u^{\alpha_{1}} \ldots u^{\alpha_{d-3}} .
\end{aligned}
$$

Hence, in the classical description, each momentum must simply be replaced by the four-velocity. Now, the effective observer scalar linked to the operator $\hat{a}$ can be expressed via $\hat{A}_{*}$ and $\hat{E}_{*}$ of different dimensionalities:

$$
\begin{aligned}
\hat{A}_{*, \mathrm{eff}}^{(d)} & \equiv \Xi_{a}^{(d)} a_{\mathrm{eff}, \mu \alpha_{1} \ldots \alpha_{d-3}}^{(d)} u^{\mu} u^{\alpha_{1}} \ldots u^{\alpha_{d-3}} \\
& =\Xi_{a}^{(d)}\left(a_{\mu \alpha_{1} \ldots \alpha_{d-3}}^{(d)}-\frac{1}{m_{\psi}} \eta_{\mu \alpha_{1}} e_{\alpha_{2} \ldots \alpha_{d-3}}^{(d-1)}\right) u^{\mu} u^{\alpha_{1}} \ldots u^{\alpha_{d-3}} \\
& =\Xi_{a}^{(d)} \hat{a}_{*}^{(d)}-\frac{m_{\psi}^{d-4}}{\left(u^{2}\right)^{(d-5) / 2}} e_{\alpha_{2} \ldots \alpha_{d-3}}^{(d-1)} u^{\alpha_{2}} \ldots u^{\alpha_{d-3}} \\
& =\Xi_{a}^{(d)} \hat{a}_{*}^{(d)}-\Xi_{e}^{(d-1)} \hat{e}_{*}^{(d-1)}=\hat{A}_{*}^{(d)}-\hat{E}_{*}^{(d-1)}
\end{aligned}
$$

which is consistent with the parameters listed in Table I. An analog correspondence can be derived for the effective observer scalar related to $\hat{c}$ :

$$
\hat{C}_{*, \text { eff }}^{(d)}=\hat{C}_{*}^{(d)}-\hat{M}_{*}^{(d-1)} .
$$

Such connections enable Lagrangians to be associated with effective coefficients and at first order in Lorentz violation they are given by

$$
\begin{aligned}
& L_{\text {eff }}^{\hat{a}^{(d)}}=-m_{\psi} \sqrt{u^{2}}-\hat{A}_{*}^{(d)}+\hat{E}_{*}^{(d-1)}, \\
& L^{\hat{c}_{\mathrm{eff}}^{(d)}}=-m_{\psi} \sqrt{u^{2}}-\hat{C}_{*}^{(d)}+\hat{M}_{*}^{(d-1)} .
\end{aligned}
$$

These results already demonstrate how at leading order in Lorentz violation, Lagrangians for different component coefficients can be composed to obtain new results for combinations of coefficients. A similar procedure can be carried out for the effective coefficients associated with the spin-nondegenerate operators:

$$
\begin{aligned}
& \left.L_{\text {eff }}^{\tilde{\hat{g}}_{\text {even }}^{(d)}}\right|_{d \geq 4}=-m_{\psi} \sqrt{u^{2}} \mp \sqrt{-\tilde{\hat{G}}_{*}^{\mu} \tilde{\hat{G}}_{* \mu}+\frac{2}{\sqrt{u^{2}}}\left[\tilde{\hat{G}}_{*} \hat{B}_{*}-\left(\tilde{\hat{G}}_{*}^{\mu} \hat{B}_{* \mu}\right) u^{2}\right]+\hat{B}_{*}^{2}-\left(\hat{B}_{*}^{\mu} \hat{B}_{* \mu}\right) u^{2}} \\
& =-m_{\psi} \sqrt{u^{2}} \mp \sqrt{-\tilde{\hat{G}}_{\mathrm{eff}, *}^{\mu} \tilde{\hat{G}}_{\mathrm{eff}, * \mu}},
\end{aligned}
$$




$$
\begin{aligned}
L_{\text {feff }}^{(d>3)} & =-m_{\psi} \sqrt{u^{2}} \mp \sqrt{-\tilde{\hat{H}}_{*}^{\mu} \tilde{\hat{H}}_{* \mu}+\frac{2}{\sqrt{u^{2}}}\left[\tilde{\hat{H}}_{*} \hat{D}_{*}-\left(\tilde{\hat{H}}_{*}^{\mu} \hat{D}_{* \mu}\right) u^{2}\right]+\hat{D}_{*}^{2}-\left(\hat{D}_{*}^{\mu} \hat{D}_{* \mu}\right) u^{2}} \\
& =-m_{\psi} \sqrt{u^{2}} \mp \sqrt{-\tilde{\hat{H}}_{\mathrm{eff}, *}^{\mu} \tilde{\hat{H}}_{\mathrm{eff}, * \mu}}, \\
\tilde{\hat{G}}_{* \mu, \mathrm{eff}}^{(d)} & \equiv \Xi_{g}^{(d)} \tilde{\hat{g}}_{* \mu, \mathrm{eff}}^{(d)}, \quad \tilde{\hat{g}}_{* \mu, \mathrm{eff}}^{(d)} \equiv \tilde{g}_{\mathrm{eff}, \mu \nu \alpha_{1} \ldots \alpha_{d-3}}^{(d)} u^{\nu} u^{\alpha_{1}} \ldots u^{\alpha_{d-3}}, \\
\tilde{\hat{H}}_{* \mu, \mathrm{eff}}^{(d)} & \equiv \Xi_{H}^{(d)} \tilde{\hat{h}}_{* \mu, \mathrm{eff}}^{(d)}, \quad \tilde{\hat{h}}_{* \mu, \mathrm{eff}}^{(d)} \equiv \tilde{H}_{\mathrm{eff}, \mu \nu \alpha_{1} \ldots \alpha_{d-3}}^{(d)} u^{\nu} u^{\alpha_{1}} \ldots u^{\alpha_{d-3}} .
\end{aligned}
$$

In Eqs. (2.29a), (2.29b) the mass dimension of the effective coefficients has again been omitted for brevity. The Lagrangians for the $\hat{b}, \hat{g}$ operators are contained in Eq. (2.29a) as special cases where those for $\hat{d}, \hat{H}$ follow from Eq. (2.29b) by setting suitable coefficients to zero. Note that the pairs of coefficients even mix in the Lagrangians of this form.

Finally, the proofs for the operators $\hat{b}, \hat{H}$ shown in Appendices A, B can be taken over literally to the situation when operators of different mass dimensions are summed over. The argument is the same as that presented for the spin-degenerate operators. The only thing to do is to replace $\hat{X}_{*}^{(d)}$ by a suitable sum, i.e., $\hat{X}_{*}^{(d)} \mapsto \sum_{d} \hat{X}_{*}^{(d)}$ where the summation runs over all $d$ permitted. The latter sum is then still positively homogeneous of degree 1 in the velocity, as the individual contributions are. Note that $\hat{\mathcal{X}}_{*}$ depends on bilinear combinations of $\hat{X}_{*}$, i.e., summations over the mass dimension are carried out under the square root and not in front of it. By doing so, coefficients of different mass dimensions may mix.

\section{GENERAL FIRST-ORDER LAGRANGIAN OF SME FERMION SECTOR}

Comparing the previously obtained Lagrangians to the corresponding first-order dispersion relations reveals plenty of similarities. Therefore, we found that there exists a direct map from the dispersion relation $E^{( \pm)}$to the associated classical Lagrangians $L^{( \pm)}$at first order in Lorentz violation. Consider the first-order dispersion relation of the nonminimal SME that is known to be of the form

$$
\begin{aligned}
E^{( \pm)} & =E_{0}-\frac{1}{E_{0}}\left(p \cdot \hat{\mathcal{V}}_{\text {eff }} \mp \Upsilon\right), \\
\Upsilon & =\sqrt{p^{\mu}\left(\tilde{\hat{\mathcal{T}}}_{\text {eff }}\right)_{\mu \varrho}\left(\tilde{\hat{\mathcal{T}}}_{\text {eff }}\right)^{\varrho}{ }_{\nu} p^{\nu},}
\end{aligned}
$$

with the effective operators transformed to momentum space

$\hat{\mathcal{V}}_{\text {eff }}^{\mu} \equiv \hat{\mathcal{V}}^{\mu}+\frac{1}{m_{\psi}} p^{\mu} \hat{\mathcal{S}}, \quad \tilde{\hat{\mathcal{T}}}_{\text {eff }}^{\mu \nu} \equiv \tilde{\hat{\mathcal{T}}}^{\mu \nu}+\frac{1}{m_{\psi}} p^{[\mu} \hat{\mathcal{A}}^{\nu]}$, cf. Eq. (43) in [23]. The map leading directly from the dispersion relation to the classical Lagrangian involves the following steps. First, perform the replacement $E_{0} \mapsto$ $-m_{\psi} \sqrt{u^{2}}$. Second, carry out $p_{\mu} \mapsto m_{\psi} u_{\mu} / \sqrt{u^{2}}$ in the Lorentz-violating term. Third, multiply the Lorentz-violating contribution by $u^{2}$ to ensure positive homogeneity of first degree in the velocity. An analog map was found in [30] within the minimal SME where its validity was demonstrated to second order in the velocity and momentum only. The procedure previously described is a generalization that is valid in the nonminimal SME and at all orders in the velocity and momentum. Applying this map to the dispersion relation of Eq. (3.1), produces the first-order classical "master" Lagrangian including all operators of the nonminimal SME:

$$
\begin{gathered}
L_{\text {master }}^{( \pm)}=-m_{\psi} \sqrt{u^{2}}+\hat{\mathcal{V}}_{*, \text { eff }} \mp \Upsilon_{*}, \\
\Upsilon_{*}=\sqrt{-\left(\tilde{\hat{\mathcal{T}}}_{*, \mathrm{eff}}\right)^{\mu}\left(\tilde{\hat{\mathcal{T}}}_{*, \mathrm{eff}}\right)_{\mu},}
\end{gathered}
$$

with a slew of observer scalars and (pseudo)vectors that correspond to the effective operators considered:

$$
\begin{aligned}
\hat{\mathcal{V}}_{*, \text { eff }} & \equiv \hat{\mathcal{V}}_{*}+\sqrt{u^{2}} \hat{\mathcal{S}}_{*}, \\
\tilde{\hat{\mathcal{T}}}_{* \text {,eff }}^{\mu} & \equiv \tilde{\hat{\mathcal{T}}}_{*}^{\mu}+\frac{u^{\mu}}{\sqrt{u^{2}}} \hat{\mathcal{A}}_{*}-\sqrt{u^{2}} \hat{\mathcal{A}}_{*}^{\mu}, \\
\hat{\mathcal{S}}_{*} & \equiv \sum_{d \geq 4, \text { even }} \Xi_{\mathcal{V}}^{(d)}\left(\hat{e}_{*}^{(d)}-\frac{m_{\psi}}{\sqrt{u^{2}}} \hat{m}_{*}^{(d+1)}\right), \\
\hat{\mathcal{V}}_{* \mu} & \equiv \sum_{d \geq 3, \mathrm{odd}} \Xi_{\mathcal{V}}^{(d)}\left(\frac{m_{\psi}}{\sqrt{u^{2}}} \hat{c}_{* \mu}^{(d+1)}-\hat{a}_{* \mu}^{(d)}\right), \\
\hat{\mathcal{V}}_{*} & \equiv \hat{\mathcal{V}}_{* \mu} u^{\mu}, \quad \Xi_{\mathcal{V}}^{(d)}=\frac{m_{\psi}^{d-3}}{\left(u^{2}\right)^{(d-3) / 2}}, \\
\hat{\mathcal{A}}_{* \mu} & \equiv \sum_{d \geq 3, \mathrm{odd}} \Xi_{\mathcal{A}}^{(d)}\left(\frac{m_{\psi}}{\sqrt{u^{2}}} \hat{d}_{* \mu}^{(d+1)}-\hat{b}_{* \mu}^{(d)}\right), \\
\hat{\mathcal{A}}_{*} & \equiv \hat{\mathcal{A}}_{* \mu} u^{\mu}, \quad \Xi_{\mathcal{A}}^{(d)}=\frac{m_{\psi}^{d-3}}{\left(u^{2}\right)^{(d-3) / 2}}, \\
\tilde{\hat{\mathcal{T}}}_{* \mu} & \equiv \sum_{d \geq 3, \mathrm{odd}} \Xi_{\mathcal{A}}^{(d)}\left(\frac{m_{\psi}}{\sqrt{u^{2}}} \tilde{\hat{g}}_{* \mu}^{(d+1)}-\tilde{\hat{H}}_{* \mu}^{(d)}\right) .
\end{aligned}
$$


Several remarks are in order. First, the Lagrangians associated with the spin-degenerate operators are completely governed by $\hat{\mathcal{V}}_{* \text {,eff }}$, whereas the spin-nondegenerate results are described by $\tilde{\hat{\mathcal{T}}}_{* \text {,eff. }}$ Second, all Lagrangians found previously are contained in the latter general result. Third, the two signs before the spin-nondegenerate contribution are switched when comparing the dispersion relations to the Lagrangians. Fourth, the only Lagrangian that is not directly contained in this general result is that for the $f$ coefficients. Since the Lagrangian, as it stands, is valid at first order in Lorentz violation only, we do not intend to add the Lagrangian for $\hat{f}$, as the latter coefficients do not provide a linear contribution. However, recall that $f_{*}^{(d)}$ squared can just be mapped onto $c_{*}^{(d)}$, cf. Eqs. (2.22).

The proof that the latter Lagrangian fulfills Eqs. (2.1) can be put together from the proofs previously carried out. The Lagrangian for $\hat{\mathcal{T}}_{* \text {,eff }}=0$ is that of the spin-degenerate operators making the corresponding proof of Sec. II applicable. For $\hat{\mathcal{V}}_{* \text {,eff }}=0$ we can take over the proof for $\hat{H}$ of Appendix $\mathrm{B}$, as the Lagrangian for the $H$ coefficients is exactly of this form. Each contribution is of first order in Lorentz violation, which is why both proofs can be combined. The spin-degenerate and spin-nondegenerate operators do not mix with each other at this level of approximation, after all.

\section{CONCLUSIONS}

In the current paper, we derived the leading-order classical Lagrangian covering all operators of the nonminimal SME fermion sector. The result for a particular operator can be obtained from this master Lagrangian by setting all other coefficients to zero. The Lagrangian for each operator was found to be a natural generalization of the already known minimal results where the minimal coefficients are replaced by the infinite sum over all nonminimal coefficients appropriately contracted with four-velocities. Furthermore, each Lorentz-violating contribution is multiplied by a factor ensuring both consistency of the mass dimension and positive homogeneity of first degree in the velocity. The first-order Lagrangian shares a lot of similarities with the corresponding dispersion relations. The modified terms for the spin-degenerate operators are directly proportional to sums of the coefficients suitably contracted with four-velocities. In contrast, the Lorentzviolating terms linked to the spin-nondegenerate operators involve a square root of a bilinear combination of controlling coefficients and four-velocities. Terms of these shapes can be considered as generalizations of structures that are known as bipartite in the literature.

With the master Lagrangian at hand, the description of Lorentz violation for classical systems should now be feasible for any kind of Lorentz-violating operator. The only caveat is that the Lagrangian obtained is the leading-order result only. However, as Lorentz violation is perturbative, the result should at least be sufficient for phenomenological studies. Terms of higher order in the perturbative expansion are supposedly much more involved, as suitable observer scalars can now be formed from component coefficients of different type and mass dimension. In future works, we intend to investigate whether the Lagrangians found can be promoted to Finsler structures. If this turns out to be possible, the properties of these Finsler structures will be an interesting topic to investigate both for physicists and mathematicians.

\section{ACKNOWLEDGMENTS}

It is a pleasure to thank V. A. Kostelecký for suggestions on an early draft of the paper. Also, the authors are grateful to the anonymous referee for several useful remarks sharpening certain aspects of the first version of the paper. The current work was financially supported by FAPEMA via the Grant No. FAPEMA/Pos-grad/03978/15.

\section{APPENDIX A: ANALYTICAL PROOF FOR THE $b$ COEFFICIENTS}

In this part of the Appendix, we would like to demonstrate that the first-order classical Lagrangian of Eq. (2.15) satisfies the defining Eqs. (2.1) of the map from the fieldtheory description to the classical point-particle analog. The calculation will be carried out by analytical means where higher-order terms will be discarded. The Lorentzviolating contribution is positively homogeneous of first degree in the velocity, which will turn out to be very helpful. Before starting with the proof, recall the classical Lagrangian found for the $b$ coefficients:

$$
\begin{aligned}
L^{\hat{b}^{(d)}} & =-m_{\psi} \sqrt{u^{2}} \mp \hat{\mathcal{B}}_{*}, \\
\hat{\mathcal{B}}_{*} & =\sqrt{\hat{B}_{*}^{2}-\left(\hat{B}_{*}^{\mu} \hat{B}_{* \mu}\right) u^{2}} .
\end{aligned}
$$

\section{Dispersion equation}

The first and undoubtedly easier task is to check the validity of Eq. (2.1a) for the canonical momentum. The latter can be cast into the form

$$
p_{\mu}=-\frac{\partial L^{\hat{b}^{(d)}}}{\partial u^{\mu}}=m_{\psi} \frac{u_{\mu}}{\sqrt{u^{2}}} \pm \frac{\partial \hat{\mathcal{B}}_{*}}{\partial u^{\mu}} .
$$

In the Lorentz-violating operator it suffices to replace each momentum by the leading-order term of the previous expression such that $\hat{b}_{\mu} \approx \Xi_{b} \hat{b}_{* \mu}$. The left-hand side of the general dispersion equation has the form

$$
\mathcal{R}=\left(p^{2}-m_{\psi}^{2}\right)^{2}+4\left[\hat{b}^{2} m_{\psi}^{2}-(\hat{b} \cdot p)^{2}\right]+\cdots .
$$

The leading-order Lorentz-violating terms in the dispersion equation are of second order, which is why these contributions have to cancel each other when the classical 
Lagrangian is supposed to be valid. Hence, all expressions must be computed at second order in Lorentz violation and higher-order terms are discarded. The square of the canonical momentum is given by

$$
\begin{aligned}
p^{2} & =m_{\psi}^{2} \pm \frac{2 m_{\psi}}{\sqrt{u^{2}}} u^{\mu} \frac{\partial \hat{\mathcal{B}}_{*}}{\partial u^{\mu}}+\left(\frac{\partial \hat{\mathcal{B}}_{*}}{\partial u}\right)^{2} \\
& =m_{\psi}^{2} \pm \frac{2 m_{\psi}}{\sqrt{u^{2}}} \hat{\mathcal{B}}_{*}+\left(\frac{\partial \hat{\mathcal{B}}_{*}}{\partial u}\right)^{2},
\end{aligned}
$$

where we used Euler's theorem for $\hat{\mathcal{B}}_{*}$ in the form

$$
u^{\mu} \frac{\partial \hat{\mathcal{B}}_{*}}{\partial u^{\mu}}=\hat{\mathcal{B}}_{*} .
$$

The remaining terms in the dispersion equation at second order in Lorentz violation are written as follows:

$$
\begin{aligned}
\hat{b} \cdot p & =m_{\psi} \frac{\hat{B}_{*}}{\sqrt{u^{2}}} \pm \hat{B}_{*}^{\mu} \frac{\partial \hat{\mathcal{B}}_{*}}{\partial u^{\mu}}+\cdots, \\
(\hat{b} \cdot p)^{2} & =m_{\psi}^{2} \frac{\hat{B}_{*}^{2}}{u^{2}}+\cdots
\end{aligned}
$$

Inserting these results into the dispersion equation, all of the second-order terms in Lorentz violation compensate each other:

$$
\begin{aligned}
\mathcal{R} & =\left( \pm \frac{2 m_{\psi}}{\sqrt{u^{2}}} \hat{\mathcal{B}}_{*}\right)^{2}+4\left(\hat{b}^{2} m_{\psi}^{2}-m_{\psi}^{2} \frac{\hat{B}_{*}^{2}}{u^{2}}\right)+\cdots \\
& =\frac{4 m_{\psi}^{2}}{u^{2}} \hat{\mathcal{B}}_{*}^{2}+4 m_{\psi}^{2}\left(\hat{B}_{*}^{\mu} \hat{B}_{* \mu}-\frac{\hat{B}_{*}^{2}}{u^{2}}\right)=0,
\end{aligned}
$$

according to Eq. (A1b). This outcome demonstrates that the canonical momentum based on the classical Lagrangian satisfies the dispersion equation at the order desired.

\section{Velocity correspondence}

In this paragraph we would like to demonstrate the validity of Eq. (2.1b) for the classical Lagrangian under consideration. As the general formula for the dispersion relation may be complicated, we compute the first (implicit) derivative of the dispersion equation and replace each $\partial p_{0} / \partial p_{i}$ by $-u^{i} / u^{0}$. For the classical Lagrangian to be valid, all contributions at second order in Lorentz violation have to compensate each other. It is reasonable to split the implicit derivative into three parts as follows:

$$
\begin{aligned}
\frac{\partial \mathcal{R}}{\partial p_{i}} & =\left.\sum_{l=1 \ldots 3} \frac{\partial \mathcal{R}}{\partial p_{i}}\right|^{(l)}, \\
\left.\frac{\partial \mathcal{R}}{\partial p_{i}}\right|^{(1)} & =-4\left(p^{2}-m_{\psi}^{2}\right)\left(\frac{u^{i}}{u^{0}} p_{0}+p_{i}\right), \\
\left.\frac{\partial \mathcal{R}}{\partial p_{i}}\right|^{(2)} & =8(\hat{b} \cdot p)\left(\frac{u^{i}}{u^{0}} \hat{b}_{0}+\hat{b}_{i}\right),
\end{aligned}
$$

$$
\left.\frac{\partial \mathcal{R}}{\partial p_{i}}\right|^{(3)}=8 m_{\psi}^{2} \hat{b}^{\nu} \frac{\partial \hat{b}_{\nu}}{\partial p_{i}}-8(\hat{b} \cdot p) p^{\nu} \frac{\partial \hat{b}_{\nu}}{\partial p_{i}}
$$

The third contribution takes into account a possible momentum dependence of the controlling coefficients that arises for nonminimal frameworks. For the minimal $b$ coefficients, this term just vanishes. We start computing the first part:

$$
\left.\frac{\partial \mathcal{R}}{\partial p_{i}}\right|^{(1)}=-\frac{8 m_{\psi}}{\sqrt{u^{2}}} \hat{\mathcal{B}}_{*}\left(\frac{u^{i}}{u^{0}} \frac{\partial \hat{\mathcal{B}}_{*}}{\partial u^{0}}+\frac{\partial \hat{\mathcal{B}}_{*}}{\partial u^{i}}\right)+\cdots
$$

The second part of the implicit derivative can be obtained quickly, as well:

$$
\begin{aligned}
\left.\frac{\partial \mathcal{R}}{\partial p_{i}}\right|^{(2)} & =\frac{8 m_{\psi}}{\sqrt{u^{2}}}(\hat{b} \cdot u)\left(\frac{u^{i}}{u^{0}} \hat{b}_{0}+\hat{b}_{i}\right)+\cdots \\
& =\frac{8 m_{\psi}}{\sqrt{u^{2}}} \hat{B}_{*}\left(\frac{u^{i}}{u^{0}} \hat{B}_{* 0}+\hat{B}_{* i}\right) .
\end{aligned}
$$

Summing the two contributions obtained leads to

$$
\begin{aligned}
\left.\sum_{l=1 \ldots 2} \frac{\partial \mathcal{R}}{\partial p_{i}}\right|^{(l)}= & \frac{8 m_{\psi}}{\sqrt{u^{2}}}\left[\hat{B}_{*}\left(\frac{u^{i}}{u^{0}} \hat{B}_{* 0}+\hat{B}_{* i}\right)\right. \\
& \left.-\hat{\mathcal{B}}_{*}\left(\frac{u^{i}}{u^{0}} \frac{\partial \hat{\mathcal{B}}_{*}}{\partial u^{0}}+\frac{\partial \hat{\mathcal{B}}_{*}}{\partial u^{i}}\right)\right]+\cdots \\
= & \frac{8 m_{\psi}}{\sqrt{u^{2}}}\left\{\hat{B}_{*}\left(\frac{u^{i}}{u^{0}} \hat{B}_{* 0}+\hat{B}_{* i}\right)\right. \\
& -\left[\frac{u^{i}}{u^{0}}\left(\hat{B}_{*} \frac{\partial \hat{B}_{*}}{\partial u^{0}}-u^{2} \hat{B}_{*}^{\nu} \frac{\partial \hat{B}_{* \nu}}{\partial u^{0}}\right)\right. \\
& \left.\left.+\hat{B}_{*} \frac{\partial \hat{B}_{*}}{\partial u^{i}}-u^{2} \hat{B}_{*}^{\nu} \frac{\partial \hat{B}_{* \nu}}{\partial u^{i}}\right]\right\}
\end{aligned}
$$

where we used

$$
\frac{\partial \hat{\mathcal{B}}_{*}}{\partial u^{\mu}}=\frac{1}{\hat{\mathcal{B}}_{*}}\left(\hat{B}_{*} \frac{\partial \hat{B}_{*}}{\partial u^{\mu}}-\hat{B}_{*}^{\nu} \hat{B}_{* \nu} u_{\mu}-u^{2} \hat{B}_{*}^{\nu} \frac{\partial \hat{B}_{* \nu}}{\partial u^{\mu}}\right) .
$$

Finally, the third part provides

$$
\left.\frac{\partial \mathcal{R}}{\partial p_{i}}\right|^{(3)}=8 m_{\psi}^{2}\left(\hat{B}_{*}^{\nu}-\frac{\hat{B}_{*} u^{\nu}}{u^{2}}\right) \frac{\partial \hat{b}_{\nu}}{\partial p_{i}}+\cdots .
$$

At this point we express the derivative of the controlling coefficients with respect to the momentum as a derivative with respect to the velocity:

$$
\frac{\partial \hat{b}_{\mu}}{\partial p_{i}}=\frac{\partial \hat{b}_{\mu}}{\partial u^{\nu}} \frac{\partial u^{\nu}}{\partial p_{i}}=-\frac{\sqrt{u^{2}}}{m_{\psi}}\left(\frac{u^{i}}{u^{0}} \frac{\partial \hat{B}_{* \mu}}{\partial u^{0}}+\frac{\partial \hat{B}_{* \mu}}{\partial u^{i}}\right),
$$

where we employed Eqs. (2.10), (2.11). All second-order terms in Lorentz violation compensate each other in the implicit derivative: 


$$
\begin{aligned}
\frac{\partial \mathcal{R}}{\partial p_{i}}= & \frac{8 m_{\psi}}{\sqrt{u^{2}}}\left[\hat{B}_{*}\left(\frac{u^{i}}{u^{0}} \hat{B}_{* 0}+\hat{B}_{* i}\right)-\hat{B}_{*}\left(\frac{u^{i}}{u^{0}} \frac{\partial \hat{B}_{*}}{\partial u^{0}}+\frac{\partial \hat{B}_{*}}{\partial u^{i}}\right)\right. \\
& +u^{2}\left(\frac{u^{i}}{u^{0}} \hat{B}_{*}^{\nu} \frac{\partial \hat{B}_{* \nu}}{\partial u^{0}}+\hat{B}_{*}^{\nu} \frac{\partial \hat{B}_{* \nu}}{\partial u^{i}}\right) \\
& \left.-\left(\hat{B}_{*}^{\nu} u^{2}-\hat{B}_{*} u^{\nu}\right)\left(\frac{u^{i}}{u^{0}} \frac{\partial \hat{B}_{* \nu}}{\partial u^{0}}+\frac{\partial \hat{B}_{* \nu}}{\partial u^{i}}\right)\right]+\cdots=0 .
\end{aligned}
$$

To arrive at this result, we additionally inserted

$$
\begin{gathered}
\frac{\partial \hat{B}_{*}}{\partial u^{0}}=\frac{\partial\left(u^{\nu} \hat{\boldsymbol{B}}_{* \nu}\right)}{\partial u^{0}}=\hat{B}_{*}^{0}+u^{\nu} \frac{\partial \hat{\boldsymbol{B}}_{* \nu}}{\partial u^{0}}, \\
\frac{\partial \hat{\boldsymbol{B}}_{*}}{\partial u^{i}}=\frac{\partial\left(u^{\nu} \hat{\boldsymbol{B}}_{* \nu}\right)}{\partial u^{i}}=\hat{B}_{* i}+u^{\nu} \frac{\partial \hat{\boldsymbol{B}}_{* \nu}}{\partial u^{i}} .
\end{gathered}
$$

\section{APPENDIX B: ANALYTICAL PROOF FOR THE $H$ COEFFICIENTS}

Here we would like to carry out a proof analog to that for the $b$ coefficients. As a reminder, the classical Lagrangian found for the operator $\hat{H}$ is given by

$$
\begin{aligned}
L^{\hat{H}^{(d)}} & =-m_{\psi} \sqrt{u^{2}} \mp \hat{\mathcal{H}}_{*}, \\
\hat{\mathcal{H}}_{*} & =\sqrt{-\tilde{\hat{H}}_{*}^{\mu} \tilde{\hat{H}}_{* \mu}} .
\end{aligned}
$$

\section{Dispersion equation}

First of all, it must be shown that the canonical momentum satisfies Eq. (2.1a). Its form is completely analog to that for the $b$ coefficients:

$$
p_{\mu}=-\frac{\partial L^{\hat{H}^{(d)}}}{\partial u^{\mu}}=m_{\psi} \frac{u_{\mu}}{\sqrt{u^{2}}} \pm \frac{\partial \hat{\mathcal{H}}_{*}}{\partial u^{\mu}} .
$$

Replacing each momentum component contracted with the $H$ coefficients by the standard term $m_{\psi} u^{\mu} / u^{2}$ produces $\hat{H}_{\mu \nu} \approx \Xi_{H} \hat{H}_{* \mu \nu}$. Neglecting all Lorentz-violating contributions beyond the second order, the dispersion equation can be expressed in the form

$$
\begin{aligned}
\mathcal{R}= & p^{4}-2 m_{\psi}^{2} p^{2}+m_{\psi}^{4}-m_{\psi}^{2} \hat{H}^{\mu \nu} \hat{H}_{\mu \nu} \\
& -2 p_{\mu}\left(\hat{H}^{\mu \nu}-\mathrm{i} \tilde{\hat{H}}^{\mu \nu}\right)\left(\hat{H}_{\nu \varrho}+\mathrm{i} \tilde{\hat{H}}_{\nu \varrho}\right) p^{\varrho}+\cdots \\
= & \left(p^{2}-m_{\psi}^{2}\right)^{2}+\left(p^{2}-m_{\psi}^{2}\right) \hat{H}^{\mu \nu} \hat{H}_{\mu \nu} \\
& -4 p^{\mu} \tilde{\hat{H}}_{\mu Q} \tilde{\hat{H}}_{\nu}{ }_{\nu} p^{\nu}+\cdots \\
= & \left(p^{2}-m_{\psi}^{2}\right)^{2}-4 p^{\mu} \tilde{\hat{H}}_{\mu \varrho} \tilde{\hat{H}}_{\nu}{ }_{\nu} p^{\nu}+\cdots
\end{aligned}
$$

because of the helpful relationship

$$
\begin{aligned}
& p_{\mu}\left(\hat{H}^{\mu \nu}-\mathrm{i} \tilde{\hat{H}}^{\mu \nu}\right)\left(\hat{H}_{\nu \varrho}+\mathrm{i} \tilde{\hat{H}}_{\nu \varrho}\right) p^{\varrho} \\
& =2 p^{\mu} \tilde{\hat{H}}_{\mu \varrho} \tilde{\hat{H}}_{\nu}{ }_{\nu} p^{\nu}-\frac{1}{2} p^{2} \hat{H}^{\mu \nu} \hat{H}_{\mu \nu} .
\end{aligned}
$$

The four-momentum squared reads

$$
\begin{aligned}
p^{2} & =m_{\psi}^{2} \pm \frac{2 m_{\psi}}{\sqrt{u^{2}}} u^{\mu} \frac{\partial \hat{\mathcal{H}}_{*}}{\partial u^{\mu}}+\left(\frac{\partial \hat{\mathcal{H}}_{*}}{\partial u}\right)^{2} \\
& =m_{\psi}^{2} \pm \frac{2 m_{\psi}}{\sqrt{u^{2}}} \hat{\mathcal{H}}_{*}+\left(\frac{\partial \hat{\mathcal{H}}_{*}}{\partial u}\right)^{2},
\end{aligned}
$$

where we exploited Euler's theorem applied to the characteristic quantity $\hat{\mathcal{H}}_{*}$ :

$$
u^{\mu} \frac{\partial \hat{\mathcal{H}}_{*}}{\partial u^{\mu}}=\hat{\mathcal{H}}_{*} .
$$

All of the ingredients are inserted into the dispersion equation showing that the second-order terms in Lorentz violation cancel each other:

$$
\mathcal{R}=\left( \pm \frac{2 m_{\psi}}{\sqrt{u^{2}}} \hat{\mathcal{H}}_{*}\right)^{2}-\frac{4 m_{\psi}^{2}}{u^{2}} \hat{\mathcal{H}}_{*}^{2}+\cdots=0 .
$$

\section{Velocity correspondence}

Now we demonstrate the validity of Eq. (2.1b). The first derivative of the dispersion equation is calculated implicitly with each $\partial p_{0} / \partial p_{i}$ replaced by $-u^{i} / u^{0}$. Due to the similarities of the dispersion equations for the $b$ and $H$ coefficients, we again split the derivative into three parts. The first two of these are obtained in total analogy to those for the $b$ coefficients:

$$
\begin{aligned}
\left.\frac{\partial \mathcal{R}}{\partial p_{i}}\right|^{(1)} & \equiv \frac{\partial p^{4}}{\partial p_{i}}=-\frac{8 m_{\psi}}{\sqrt{u^{2}}} \hat{\mathcal{H}}_{*}\left(\frac{u^{i}}{u^{0}} \frac{\partial \hat{\mathcal{H}}_{*}}{\partial u^{0}}+\frac{\partial \hat{\mathcal{H}}_{*}}{\partial u^{i}}\right)+\cdots, \\
\left.\frac{\partial \mathcal{R}}{\partial p_{i}}\right|^{(2)} & \equiv 8\left(\tilde{\hat{H}}_{\varrho \mu} p^{\mu}\right) \tilde{\hat{H}}^{\rho \nu} \frac{\partial p_{\nu}}{\partial p_{i}} \\
& =\frac{8 m_{\psi}}{\sqrt{u^{2}}} \tilde{\hat{H}}_{\varrho \mu} u^{\mu}\left(-\frac{u^{i}}{u^{0}} \tilde{\hat{H}}_{0}^{\varrho}-\tilde{\hat{H}}_{i}{ }_{i}\right) \\
& =-\frac{8 m_{\psi}}{\sqrt{u^{2}}} \tilde{\hat{H}}_{* Q}\left(\frac{u^{i}}{u^{0}} \tilde{\hat{H}}_{* 0}^{Q}+\tilde{\hat{H}}_{* i}^{Q}\right)+\cdots .
\end{aligned}
$$

Summing the latter contributions produces

$$
\begin{aligned}
\left.\sum_{l=1 \ldots 2} \frac{\partial \mathcal{R}}{\partial p_{i}}\right|^{(l)}= & -\frac{8 m_{\psi}}{\sqrt{u^{2}}}\left[\tilde{\hat{H}}_{* e}\left(\frac{u^{i}}{u^{0}} \tilde{\hat{H}}_{* 0}^{Q}+\tilde{\hat{H}}_{* i}^{Q}\right)\right. \\
& \left.+\hat{\mathcal{H}}_{*}\left(\frac{u^{i}}{u^{0}} \frac{\partial \hat{\mathcal{H}}_{*}}{\partial u^{0}}+\frac{\partial \hat{\mathcal{H}}_{*}}{\partial u^{i}}\right)\right] \\
= & \frac{8 m_{\psi}}{\sqrt{u^{2}}} \tilde{\hat{H}}_{* e}\left[\frac{u^{i}}{u^{0}}\left(\frac{\partial \tilde{\hat{H}}_{*}^{e}}{\partial u^{0}}-\tilde{\hat{H}}_{* 0}^{e}\right)+\frac{\partial \tilde{\hat{H}}_{*}^{e}}{\partial u^{i}}-\tilde{\hat{H}}_{* i}^{Q}\right] \\
& +\cdots,
\end{aligned}
$$

where the derivative of the quantity $\hat{\mathcal{H}}_{*}$ was used:

$$
\frac{\partial \hat{\mathcal{H}}_{*}}{\partial u^{\mu}}=-\frac{1}{\hat{\mathcal{H}}_{*}} \frac{\partial \tilde{\hat{H}}_{*}^{\nu}}{\partial u^{\mu}} \tilde{\hat{H}}_{* \nu} .
$$


The third part, which vanishes within the minimal SME fermion sector, contains derivatives of the Lorentzviolating operators with respect to the momentum:

$$
\begin{aligned}
& \left.\frac{\partial \mathcal{R}}{\partial p_{i}}\right|^{(3)}=-4 p^{\mu} \frac{\partial \tilde{\hat{H}}_{\mu}{ }^{e} \tilde{\hat{H}}_{Q \nu}}{\partial p_{i}} p^{\nu} \\
& =-4\left(p^{\mu} \frac{\partial \tilde{\hat{H}}_{\mu}{ }^{Q}}{\partial p_{i}} \tilde{\hat{H}}_{\varrho \nu} p^{\nu}+p^{\mu} \tilde{\hat{H}}_{\mu Q} \frac{\partial \tilde{\hat{H}}^{\varrho}{ }_{\nu}}{\partial p_{i}} p^{\nu}\right) \\
& =4\left\{\frac{\partial \tilde{\hat{H}}^{Q}}{\partial p^{i}} \tilde{\hat{H}}_{Q}-\left[\tilde{\hat{H}}^{Q}{ }_{0}\left(-\frac{u^{i}}{u^{0}}\right)-\tilde{\hat{H}}_{i}{ }_{i}\right] \tilde{\hat{H}}_{Q}+\tilde{\hat{H}}_{Q} \frac{\partial \tilde{\hat{H}}^{Q}}{\partial p^{i}}-\tilde{\hat{H}}_{Q}\left[\tilde{\hat{H}}_{0}{ }_{0}\left(-\frac{u^{i}}{u^{0}}\right)-\tilde{\hat{H}}^{Q}{ }_{i}\right]\right\} \\
& =8 \tilde{\hat{H}}_{Q}\left[\frac{\partial \tilde{\hat{H}}^{Q}}{\partial p^{i}}+\frac{u^{i}}{u^{0}} \tilde{\hat{H}}^{Q}{ }_{0}+\tilde{\hat{H}}^{Q}{ }_{i}\right] \\
& =\frac{8 m_{\psi}}{\sqrt{u^{2}}} \tilde{\hat{H}}_{* \varrho}\left[\frac{u^{i}}{u^{0}}\left(\tilde{\hat{H}}_{* 0}^{Q}-\frac{\partial \tilde{\hat{H}}_{*}^{Q}}{\partial u^{0}}\right)+\tilde{\hat{H}}_{* i}^{Q}-\frac{\partial \tilde{\hat{H}}_{*}^{Q}}{\partial u^{i}}\right]+\cdots
\end{aligned}
$$

In the final step, the derivative by the momentum was again expressed as a derivative with respect to the four-velocity:

$$
\frac{\partial \tilde{\hat{H}}_{\mu}}{\partial p_{i}}=\frac{\partial \tilde{\hat{H}}_{\mu}}{\partial u^{\sigma}} \frac{\partial u^{\sigma}}{\partial p_{i}}=-\left(\frac{u^{i}}{u^{0}} \frac{\partial \tilde{\hat{H}}_{* \mu}}{\partial u^{0}}+\frac{\partial \tilde{\hat{H}}_{* \mu}}{\partial u^{i}}\right) .
$$

In addition, Eqs. (2.10), (2.11) were employed. Now we see quickly that the sum of the first two parts equals the negative of the third, which makes all second-order terms in Lorentz violation cancel each other.

[1] V. A. Kostelecký and S. Samuel, Spontaneous breaking of Lorentz symmetry in string theory, Phys. Rev. D 39, 683 (1989).

[2] V. A. Kostelecký and S. Samuel, Phenomenological Gravitational Constraints on Strings and Higher-Dimensional Theories, Phys. Rev. Lett. 63, 224 (1989).

[3] V. A. Kostelecký and S. Samuel, Gravitational phenomenology in higher-dimensional theories and strings, Phys. Rev. D 40, 1886 (1989).

[4] V. A. Kostelecký and R. Potting, CPT and strings, Nucl. Phys. B359, 545 (1991).

[5] V. A. Kostelecký and R. Potting, $C P T$, strings, and meson factories, Phys. Rev. D 51, 3923 (1995).

[6] R. Gambini and J. Pullin, Nonstandard optics from quantum space-time, Phys. Rev. D 59, 124021 (1999).

[7] M. Bojowald, H. A. Morales-Técotl, and H. Sahlmann, Loop quantum gravity phenomenology and the issue of Lorentz invariance, Phys. Rev. D 71, 084012 (2005).

[8] G. Amelino-Camelia and S. Majid, Waves on noncommutative spacetime and gamma-ray bursts, Int. J. Mod. Phys. A 15, 4301 (2000).

[9] S. M. Carroll, J. A. Harvey, V. A. Kostelecký, C. D. Lane, and T. Okamoto, Noncommutative Field Theory and Lorentz Violation, Phys. Rev. Lett. 87, 141601 (2001).
[10] F. R. Klinkhamer and C. Rupp, Spacetime foam, $C P T$ anomaly, and photon propagation, Phys. Rev. D 70, 045020 (2004).

[11] S. Bernadotte and F. R. Klinkhamer, Bounds on length scales of classical spacetime foam models, Phys. Rev. D 75, 024028 (2007).

[12] S. Hossenfelder, Theory and phenomenology of space-time defects, Adv. High Energy Phys. 2014, 1 (2014).

[13] F. R. Klinkhamer, Z-string global gauge anomaly and Lorentz non-invariance, Nucl. Phys. B535, 233 (1998).

[14] F. R. Klinkhamer, A CPT anomaly, Nucl. Phys. B578, 277 (2000).

[15] F. R. Klinkhamer and J. Schimmel, CPT anomaly: a rigorous result in four dimensions, Nucl. Phys. B639, 241 (2002).

[16] K. J. B. Ghosh and F. R. Klinkhamer, Anomalous Lorentz and $C P T$ violation from a local Chern-Simons-like term in the effective gauge-field action, Nucl. Phys. B926, 335 (2018).

[17] P. Hořava, Quantum gravity at a Lifshitz point, Phys. Rev. D 79, 084008 (2009).

[18] D. Colladay and V. A. Kostelecký, CPT violation and the standard model, Phys. Rev. D 55, 6760 (1997). 
[19] D. Colladay and V. A. Kostelecký, Lorentz-violating extension of the standard model, Phys. Rev. D 58, 116002 (1998).

[20] V. A. Kostelecký, Gravity, Lorentz violation, and the standard model, Phys. Rev. D 69, 105009 (2004).

[21] V. A. Kostelecký and M. Mewes, Electrodynamics with Lorentz-violating operators of arbitrary dimension, Phys. Rev. D 80, 015020 (2009).

[22] V. A. Kostelecký and M. Mewes, Neutrinos with Lorentzviolating operators of arbitrary dimension, Phys. Rev. D 85, 096005 (2012).

[23] V. A. Kostelecký and M. Mewes, Fermions with Lorentzviolating operators of arbitrary dimension, Phys. Rev. D 88, 096006 (2013).

[24] O. W. Greenberg, CPT Violation Implies Violation of Lorentz Invariance, Phys. Rev. Lett. 89, 231602 (2002).

[25] V. A. Kostelecký and N. Russell, Data tables for Lorentz and CPT violation, Rev. Mod. Phys. 83, 11 (2011).

[26] V. A. Kostelecký and N. Russell, Classical kinematics for Lorentz violation, Phys. Lett. B 693, 443 (2010).

[27] D. Colladay and P. McDonald, Classical Lagrangians for momentum dependent Lorentz violation, Phys. Rev. D 85, 044042 (2012).

[28] N. Russell, Finsler-like structures from Lorentz-breaking classical particles, Phys. Rev. D 91, 045008 (2015).

[29] M. Schreck, Classical kinematics for isotropic, minimal Lorentz-violating fermion operators, Phys. Rev. D 91, 105001 (2015).
[30] M. Schreck, From classical Lagrangians to Hamilton operators in the standard model extension, Phys. Rev. D 94, 025019 (2016).

[31] M. Schreck, Eikonal approximation, Finsler structures, and implications for Lorentz-violating photons in weak gravitational fields, Phys. Rev. D 92, 125032 (2015).

[32] M. Schreck, Classical kinematics and Finsler structures for nonminimal Lorentz-violating fermions, Eur. Phys. J. C 75, 187 (2015).

[33] M. Schreck, Classical Lagrangians and Finsler structures for the nonminimal fermion sector of the standard model extension, Phys. Rev. D 93, 105017 (2016).

[34] V. A. Kostelecký, Riemann-Finsler geometry and Lorentzviolating kinematics, Phys. Lett. B 701, 137 (2011).

[35] J. Foster and R. Lehnert, Classical-physics applications for Finsler $b$ space, Phys. Lett. B 746, 164 (2015).

[36] D. Colladay and P. McDonald, Singular Lorentz-violating Lagrangians and associated Finsler structures, Phys. Rev. D 92, 085031 (2015).

[37] D. Colladay, Extended hamiltonian formalism and Lorentzviolating lagrangians, Phys. Lett. B 772, 694 (2017).

[38] V. A. Kostelecký, N. Russell, and R. Tso, Bipartite Riemann-Finsler geometry and Lorentz violation, Phys. Lett. B 716, 470 (2012).

[39] B. Altschul, Eliminating the $C P T$-odd $f$ coefficient from the Lorentz-violating standard model extension, J. Phys. A 39, 13757 (2006). 